\title{
Food sources of free sugars in children's diet and identification of lifestyle patterns associated with free sugars intake: the GRECO (Greek Childhood Obesity) study
}

\author{
Paul Farajian ${ }^{1}$, Grigoris Risvas ${ }^{1}$, Demosthenes B Panagiotakos ${ }^{2}$ and Antonis Zampelas 1,3,* \\ 'Unit of Human Nutrition, Department of Food Science and Human Nutrition, Agricultural University of Athens, lera \\ Odos 75, 11855 Athens, Greece: ${ }^{2}$ Department of Science of Dietetics - Nutrition, Harokopio University, Athens, \\ Greece: ${ }^{3}$ Department of Nutrition and Health, United Arab Emirates University, Al Ain, United Arab Emirates
}

Submitted 30 June 2015: Final revision received 5 0ctober 2015: Accepted 12 October 2015: First published online 23 November 2015

\begin{abstract}
Objective: Excessive free sugars consumption has a possible role in health issues, diet quality and obesity development. The present cross-sectional study aimed to identify the major food sources of free sugars in Greek children's diet and investigate possible associations of dietary patterns with free sugars intake.

Design: Anthropometric measurements and information on dietary and physical activity habits were obtained. Energy and free sugars intake coming from foods were estimated and principal components analysis was applied to identify dietary patterns.

Setting: The GRECO (Greek Childhood Obesity) study.

Subjects: Nationwide sample of 3089 children (aged 10-12 years).

Results: Adopting WHO criteria, $44.2 \%$ of participants were categorized as having free sugars intake above $10 \%$ of total energy intake. Mean contribution of free sugars to energy intake was $11.2 \%$, and the major food sources of free sugars differed from those of other childhood populations. Free sugars intake was not associated with overweight/obesity. Multiple linear regression analysis revealed that two lifestyle and dietary patterns, characterized by higher consumption of sweets, fast foods, fries, sugared drinks, frequently ordering/eating outside home and having meals in front of a screen (pattern 1) and higher consumption of whole fruits, $100 \%$ fruit juices, vegetables, legumes and honey/jam (pattern 2), were positively associated with free sugars intake.

Conclusions: A large proportion of children exceeded the recommended cut-off and free sugars intake was associated with lifestyle patterns rather than single foods. Public health programmes aiming to reduce free sugars consumption should be tailored on promoting the correct dietary habits of specific childhood populations.
\end{abstract}

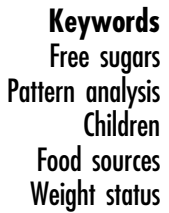

Keywords

Free sugars

Children

Weight status
Free sugars consumption has been a major issue in public health, primarily because of the possible role of foods high in free sugars in displacing intakes of nutrient-rich foods and thus diluting micronutrients in the diet ${ }^{(1-3)}$, the linkage between sugars and dental caries development ${ }^{(4)}$ and the possible contribution of excessive free sugars intake to the obesity (OB) epidemic ${ }^{(2,5)}$. Especially for children and adolescents, ensuring nutrient-dense diets is critical for optimal growth and early prevention of several chronic diseases. Following the worldwide trend revealing a considerable increase in overweight (OW) and $\mathrm{OB}$ prevalence during childhood in developed countries ${ }^{(6,7)}$, recent studies in Greek populations show very high rates of childhood OW and OB and aggravating dietary and lifestyle habits ${ }^{(8,9)}$. The potential link between excessive intake of free sugars, $\mathrm{OB}$ and other health outcomes, including diabetes and CVD risk factor alterations, has been pointed out as an important public health matter, not only in adults but in adolescents as well ${ }^{(10-13)}$.

Studies on the effects of sugar consumption on weight status or gain have shown mixed results. An inverse correlation between total sugar intake and BMI has been reported in children and adults ${ }^{(14-16)}$, while in the metaanalyses of Te Morenga et al. ${ }^{(5)}$ the assessment of cohort studies that reported data for the odds of being OW at follow-up in children consuming about one serving of sugar-sweetened beverages (SSB) daily at baseline, compared with children consuming none or very little, 
showed that those with higher intakes had significantly increased risk of being OW.

Several organizations agree that sugars intake has to be reduced and have issued dietary recommendations for sugars or added sugars that vary in terms of suggested intakes or types of sugars specified ${ }^{(1,17-19)}$. Perhaps the most commonly used recommendation is the one of the $\mathrm{WHO}$ and $\mathrm{FAO}^{(19)}$ proposing that 'free sugars' (the term preferred by the WHO, and defined as all mono- and disaccharides added to foods by the manufacturer, cook and consumer, plus sugars naturally present in honey, syrup and fruit juice) should constitute $<10 \%$ of energy intake. In many studies the investigation of the associations between free sugars and health outcomes has been restricted to the consumption of SSB, although during past years several investigators have suggested to use a holistic dietary approach on disease prevention, giving much attention to pattern analysis ${ }^{(20,21)}$. Thus, instead of looking at individual nutrients or foods, pattern analysis examines the effects of overall dietary choices and nutrient consumption and possibly explains diet-outcome relationships ${ }^{(22)}$.

Consequently, the aim of the present cross-sectional study was to estimate the major sources of free sugars in the diet of Greek schoolchildren. Additionally, following the holistic dietary assessment approach of pattern analysis another purpose of the present study was to investigate how the extracted dietary and lifestyle patterns of children were associated with free sugars intake.

\section{Methods}

\section{Study sample}

Under the context of the GRECO (Greek Childhood Obesity) study, during 2009 a representative number of randomly selected public primary schools (5th and 6th grade primary-school children, 10-12 years old) were invited to participate in the study, according to a stratified sampling procedure by sex and age group, based on the population distribution in ten regions of the country. Detailed information regarding participants' data collection has been reported elsewhere ${ }^{(8,23)}$. After checking the completeness of the provided data, from the overall 4786 measured children with signed parental consent forms, a total sample of 3089 children with complete information on free sugars intake was finally included in the analyses of the present study (mean age 10.9 (sD 0.7 ) years, $53 \%$ girls). The studied sample could be considered as representative of the overall study population (i.e. the 4786 children included for analysis in the GRECO study) regarding age and BMI distributions $(P>0.05)$, as differences were not evident between the present and the overall sample. However, differences were revealed regarding sex $(P<0.05)$ and regional distribution $(P<0 \cdot 01)$.
Nutrient intake and eating behaviour assessment

Dietary assessment was based on a validated, self-reported, semi-quantitative picture-aid FFQ, consisting of fortyeight food items commonly used in the local Greek cuisine ${ }^{(24,25)}$. All participants were asked about their usual frequency of consumption of the food items with the following response categories: 'every day', '3-6 times/ week', '2 times/week', '1 time/week', '1-2 times/month' and 'seldom/never'. The questionnaire included supplementary questions assessing the frequency of breakfast consumption and eating occasions (number of meals and snacks during the day), as well as the frequency of having meals in front of a screen (when watching television (TV)/digital video disks (DVD)/videos and/or using a games console/computer) and the frequency of having meals together with the whole family or at least one family member. Details on the type of foods consumed were also asked (such as whole-wheat bread $v$. white bread, brown rice $v$. white, whole-wheat pasta $v$. white, whole-wheat breakfast cereals $v$. plain, etc.). To estimate the daily energy and free sugars intakes coming from foods, the Nutrition Data System for Research (NDSR) software version 2011, developed by the Nutrition Coordinating Center at the University of Minnesota (Minneapolis, MN, USA) was used, as well as local food composition tables ${ }^{(26)}$.

\section{Estimation of energy under-reporting}

Energy under-reporting was estimated using the Goldberg equation $^{(27)}$ according to the ratio of energy intake (EI) to BMR. In particular, Schofield's age-specific equations were used to estimate BMR from measured weight ${ }^{(28)}$, while physical activity level was set to 1.55 indicating moderate activity according to mean values of PAQ-C score. The evaluation of individual intake considering long records was based on $28 \mathrm{~d}$ as the FFQ used in the present study referred to usual food intake of the past. The within-subject daily variation in $\mathrm{EI}\left(\mathrm{CV}_{\mathrm{wE}}\right)$ was set at $23 \%$ as a suitable average value as previously described in detail ${ }^{(29)}$, the variation in BMR $\left(\mathrm{CV}_{\mathrm{wB}}\right)$ was set at $8.5 \%$ and the between-subject variation in physical activity $\left(\mathrm{CV}_{\mathrm{tP}}\right)$ was set at $15 \%$ as suitable averages ${ }^{(27)}$. According to these, under-reporters of EI were defined as those with EI:BMR $<1.09$.

\section{Anthropometric measurements}

Body weight was measured to the nearest $0 \cdot 1 \mathrm{~kg}$ with the use of a digital scale (Tanita TBF 300). Standing height was measured using a portable stadiometer (Leicester height measure) to the nearest $0.1 \mathrm{~cm}$ without shoes. BMI $\left(\mathrm{kg} / \mathrm{m}^{2}\right)$ was calculated by dividing weight by standing height squared. OB and OW among children were calculated using the International Obesity Task Force age- and sexspecific BMI cut-off criteria ${ }^{(30)}$. Waist and hip circumferences were measured to the nearest $0 \cdot 1 \mathrm{~cm}$ with the use of a non-elastic tape (Seca, Germany) and with the participant in standing position. Waist-to-hip and 
waist-to-height ratios were also calculated. All measurements were performed during morning hours.

\section{Physical activity and sedentary behaviours assessment}

Participants were asked to complete the Physical Activity Questionnaire for Older Children (PAQ-C; score 1-5) ${ }^{(31)}$. In addition, children were also asked to report the average weekday and weekend time (h/d) spent on sedentary activities and more specifically on watching TV/DVD/ videos and/or recreational usage of a games console/ computer, together defined as screen time. By combining the former two responses, mean time (h/d) spent watching TV/DVD/videos and/or recreational usage of a games console/computer was calculated.

\section{Statistical analysis}

Continuous variables that were normally distributed are presented as mean and standard deviation. Normality was evaluated using P-P plots. Associations between categorical variables were tested by the $\chi^{2}$ test. Student's $t$ test for independent samples was used to evaluate mean differences between normally distributed variables.

To obtain lifestyle patterns, factor analysis with the principal components method was applied to the food variables ${ }^{(32)}$. After converting the reported eating frequencies of foods from the semi-quantitative FFQ into servings per day, the food variables used in principal components analysis had continuous distributions representing servings per day of the food item, food group or beverage consumed. The correlation matrix of the food variables used showed that there were several correlation coefficients with absolute value $>0 \cdot 3$. The KaiserMeyer-Olkin criterion and Bartlett's test of sphericity were also used to assess the suitability of the data for principal components analysis. An overall Kaiser-Meyer-Olkin criterion close to unity suggests very good inter-correlation of variables and that the data set is suitable for principal components analysis. Moreover, based on the criterion proposed by Kaiser - i.e. the number of components that should be retained is equal to the number of eigenvalues that are greater than one, since these components explain more information than the individual food variables - it was concluded that the first four components should be extracted. Based on the principle that the component scores (loadings) are interpreted similarly to correlation coefficients, and thus higher absolute values indicate that the food variable contributes most to the construction of the component, the food components (patterns) were named according to the foods or food groups that had absolute scores $>0 \cdot 4$.

Multiple linear regression analysis was then applied to evaluate the associations between total free sugars intake and dietary patterns derived from the principal components analysis, after adjustment for several potential confounders and testing for collinearity using their first product moments. Finally, multiple logistic regression analysis was applied to evaluate the association of free sugars intake in relation to the likelihood for a child being OW/OB. Results are presented as odds ratios and their corresponding $95 \%$ confidence intervals. All reported $P$ values were based on two-sided tests. Statistical calculations were carried out using the statistical software package PASW Statistics version 18.

\section{Results}

Using the Goldberg cut-off, $27.9 \%$ of the children were classified as energy under-reporters. In order to examine the effect of under-reporting in the results of the present study, we compared the percentage of under-reporters and normal reporters exceeding the WHO suggested limit of $10 \%$ of total energy coming from free sugars ${ }^{(19)}$ and found no differences between the different groups. In addition, when examining the main food sources of free sugars, total sugars and energy in the diet of under- and normal-reporting children, no differences were observed in the classification of sources and their contribution to total sugars and energy intake. Therefore, we did not proceed to the exclusion of under-reporters from the data set and continued our analysis with the whole sample.

Among participants, $44.2 \%$ were categorized as having free sugars intake above $10 \%$ of total energy intake. Mean total free sugars intake was 63.5 (SD 46.9) g/d and was significantly higher $(P<0.001)$ among boys $(69.7$ (SD 51.6$) \mathrm{g} / \mathrm{d})$ than girls $(58.0(\mathrm{SD} 41.6) \mathrm{g} / \mathrm{d})$; however, the proportion of boys and girls exceeding the recommended cut-off of energy intake coming from free sugars did not differ significantly. The overall mean contribution of free sugars to total energy intake was 11.2 (SD 5.4) \%, without any sex differences, and free sugars accounted for $44 \%$ of total sugars. The major food sources of free sugars in the diet of Greek children are shown in Table 1, in addition to the contribution of free sugars to total sugars intake. Anthropometric and lifestyle characteristics of the participating children according to their free sugars intake classification are presented in Table 2. Specifically, children exceeding the cut-off had fewer daily meals and snacks, and also consumed meals in front of a screen (TV, video games console or computer) more often. Mean values of all anthropometric characteristics (i.e. BMI, body weight, waist and hip circumferences, waist-to-height and waist-to-hip ratios) did not differ between the two categories of children, nor did their mean energy intake. Concerning the top ten sources of energy, these are shown graphically in Fig. 1. Among the foods contributing free sugars, sweets, $100 \%$ natural fruit juice, dairy desserts and chocolate milk were also listed in the top ten energy sources of children. Breads (presented as part of the starch group in Fig. 1) contributed 7.6 (SD 7.3) \% of total energy intake. Soft drinks and sugared fruit juices when grouped 
Table 1 Consumption of free sugars from foods and food groups and their contribution to total sugars intake in a nationwide sample of 3089 children (aged 10-12 years), GRECO (Greek Childhood Obesity) study, 2009

\begin{tabular}{|c|c|c|c|c|}
\hline \multirow[b]{2}{*}{ Food source* } & \multicolumn{2}{|c|}{$\begin{array}{l}\text { Intake of added sugars } \\
(\mathrm{g} / \mathrm{d})\end{array}$} & \multicolumn{2}{|c|}{$\begin{array}{c}\% \text { of total sugars coming from } \\
\text { added sugars }\end{array}$} \\
\hline & Mean & SD & Mean & SD \\
\hline $100 \%$ natural packaged and freshly squeezed fruit juice & $17 \cdot 8$ & $16 \cdot 9$ & $12 \cdot 4$ & 9.7 \\
\hline Sweets $\dagger$ & 11.5 & $13 \cdot 3$ & $8 \cdot 3$ & $6 \cdot 8$ \\
\hline Nectar (sugared) fruit juices & $9 \cdot 2$ & $16 \cdot 7$ & $5 \cdot 7$ & 8.4 \\
\hline Chocolate milk & 4.7 & $7 \cdot 6$ & 3.4 & 4.6 \\
\hline Soft drinks & $4 \cdot 6$ & 11.0 & 3.4 & 5.9 \\
\hline Dairy desserts & 3.5 & $6 \cdot 6$ & $2 \cdot 3$ & $3 \cdot 3$ \\
\hline Biscuits & 3.2 & 5.8 & $2 \cdot 1$ & $2 \cdot 8$ \\
\hline Cereals & 3.0 & $3 \cdot 2$ & $2 \cdot 0$ & 2.5 \\
\hline Honey/jam & $2 \cdot 7$ & $3 \cdot 8$ & $2 \cdot 0$ & $2 \cdot 7$ \\
\hline Breads & 2.5 & 2.5 & $2 \cdot 0$ & $2 \cdot 2$ \\
\hline Starchy pies & 0.5 & 0.9 & 0.4 & 0.5 \\
\hline Delicatessen & 0.2 & 0.3 & $0 \cdot 1$ & 0.4 \\
\hline
\end{tabular}

*Sorted by overall contribution of free sugars.

†Sweets category includes chocolate, chocolate bars, ice cream and pastries.

Table 2 Demographic, anthropometric and lifestyle characteristics according to free sugars contribution to total energy intake in a nationwide sample of 3089 children (aged 10-12 years), GRECO (Greek Childhood Obesity) study, 2009

\begin{tabular}{|c|c|c|c|c|c|}
\hline & \multicolumn{2}{|c|}{$\begin{array}{c}\leq 10 \% \text { of energy intake } \\
\text { from free sugars }(n 1724)\end{array}$} & \multicolumn{2}{|c|}{$\begin{array}{l}>10 \% \text { of energy intake } \\
\text { from free sugars }(n 1365)\end{array}$} & \multirow[b]{2}{*}{$P^{*}$} \\
\hline & Mean or \% & SD & Mean or \% & SD & \\
\hline Sex (\%) & & & & & 0.71 \\
\hline Males & $47 \cdot 0$ & - & $46 \cdot 3$ & - & \\
\hline Females & 53.0 & - & 53.7 & - & \\
\hline Age (years) & 10.9 & 0.7 & $10 \cdot 9$ & 0.7 & 0.43 \\
\hline Total sugars $(\mathrm{g} / \mathrm{d})$ & 113.5 & $57 \cdot 3$ & $162 \cdot 4$ & $77 \cdot 6$ & $<0.001$ \\
\hline Added sugars (g/d) & 38.8 & 24.7 & $81 \cdot 1$ & 43.5 & $<0.001$ \\
\hline Energy $(\mathrm{kJ} / \mathrm{d})$ & 9213 & 4376 & 9393 & 4615 & 0.29 \\
\hline Energy (kcal/d) & 2202 & 1046 & 2245 & 1103 & 0.29 \\
\hline Weight $(\mathrm{kg})$ & $45 \cdot 8$ & 10.7 & 43.3 & 10.8 & 0.21 \\
\hline Height $(\mathrm{cm})$ & 149.8 & $7 \cdot 7$ & $149 \cdot 6$ & $7 \cdot 6$ & 0.60 \\
\hline $\mathrm{BMI}\left(\mathrm{kg} / \mathrm{m}^{2}\right)$ & 20.3 & 3.7 & $20 \cdot 1$ & 3.7 & 0.11 \\
\hline Waist circumference $(\mathrm{cm})$ & $68 \cdot 6$ & $9 \cdot 6$ & $68 \cdot 1$ & 9.5 & 0.21 \\
\hline Hip circumference $(\mathrm{cm})$ & $83 \cdot 2$ & 9.9 & $82 \cdot 8$ & $10 \cdot 0$ & 0.34 \\
\hline Waist-to-height ratio & 0.46 & 0.06 & 0.45 & 0.06 & 0.15 \\
\hline Waist-to-hip ratio & 0.82 & 0.08 & 0.83 & 0.07 & 0.60 \\
\hline BMI status (\%) & & & & & 0.13 \\
\hline Normal weight & $58 \cdot 1$ & - & 61.7 & - & \\
\hline Overweight & 30.5 & - & $27 \cdot 2$ & - & \\
\hline Obese & 11.4 & - & $11 \cdot 1$ & _ & \\
\hline PAQ-C score (range 1-5) & 2.95 & 0.59 & 2.96 & 0.61 & 0.50 \\
\hline Screen time $(\mathrm{h} / \mathrm{d})$ & $2 \cdot 20$ & 1.40 & $2 \cdot 27$ & 1.38 & 0.27 \\
\hline Studying duration $(\mathrm{h} / \mathrm{d})$ & $2 \cdot 72$ & 1.26 & 2.56 & $1 \cdot 18$ & $<0.001$ \\
\hline Having breakfast (times/week) & 4.51 & $2 \cdot 47$ & 4.51 & $2 \cdot 39$ & 0.92 \\
\hline Number of eating occasions (times/d) & $3 \cdot 20$ & $1 \cdot 28$ & $3 \cdot 11$ & $1 \cdot 22$ & 0.06 \\
\hline Having meal while watching TV or playing video games (\%) & & & & & 0.02 \\
\hline$<3$ times/week & 81.8 & - & $78 \cdot 3$ & - & \\
\hline$\geq 3$ times/week & 18.2 & - & 21.7 & - & \\
\hline Having meal with father or mother (\%) & & & & & 0.20 \\
\hline$<5$ times/week & 45.5 & - & 43.1 & - & \\
\hline$\geq 5$ times/week & 54.5 & - & $56 \cdot 9$ & - & \\
\hline Frequency of meals outside home (plus ordering out) (\%) & & & & & 0.32 \\
\hline Rare/few times per month & 67.4 & - & $65 \cdot 6$ & - & \\
\hline 1 time/week & $22 \cdot 9$ & - & $22 \cdot 0$ & - & \\
\hline 2 times/week & 7.5 & - & 9.7 & - & \\
\hline$\geq 3$ times/week & $2 \cdot 2$ & - & $2 \cdot 7$ & - & \\
\hline
\end{tabular}

PAQ-C, Physical Activity Questionnaire for Older Children; TV, television.

${ }^{*}$ The reported $P$ values were calculated using the $t$ test and the $X^{2}$ test. 


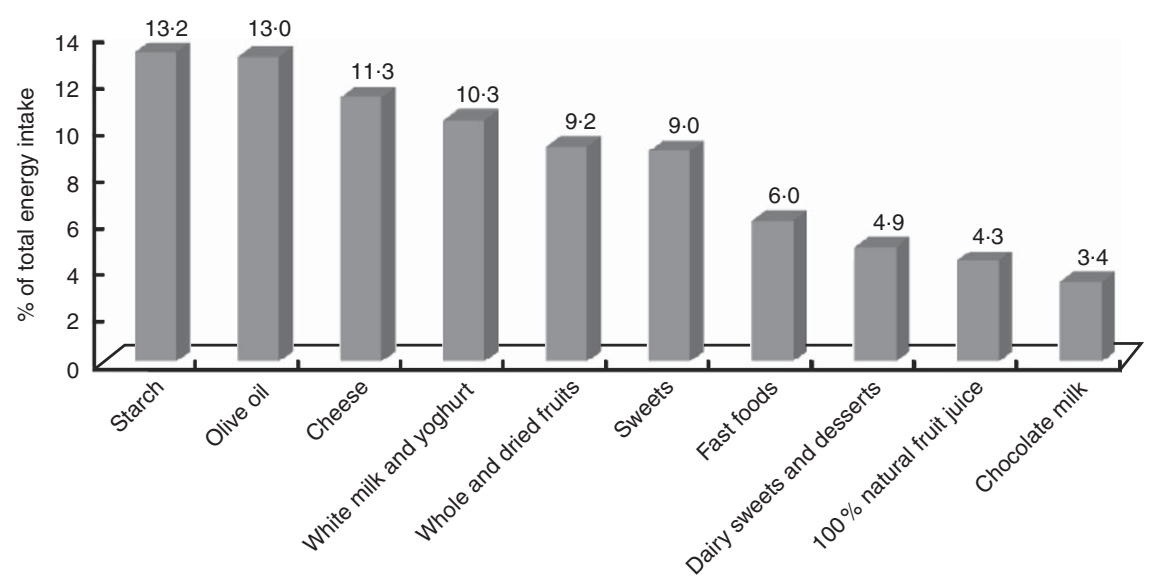

Fig. 1 Top ten sources of foods and food groups ${ }^{*}$ contributing to mean total energy intake in a nationwide sample of 3089 children (aged 10-12 years), GRECO (Greek Childhood Obesity) study, 2009. *Starch category includes breads, pasta, rice and potato (excluding breakfast cereals and French fries); cheese category includes full- and low-fat cheese; sweets category includes chocolate, chocolate bars, ice cream and pastries; fast foods category includes burgers, pizza and souvlaki (traditional food with meat); $100 \%$ natural fruit juice category includes freshly squeezed and packaged $100 \%$ fruit juice

Table 3 Score coefficients (loadings) derived from factor (principal components) analysis regarding foods or food groups consumed by the children and their lifestyle habits; nationwide sample of 3089 children (aged 10-12 years), GRECO (Greek Childhood Obesity) study, 2009

\begin{tabular}{|c|c|c|c|c|}
\hline & Factor 1 & Factor 2 & Factor 3 & Factor 4 \\
\hline Whole fruits & 0.37 & 0.48 & -0.10 & -0.27 \\
\hline $100 \%$ fruit juices* & 0.32 & 0.41 & -0.34 & -0.25 \\
\hline Vegetables & 0.26 & 0.57 & -0.02 & -0.16 \\
\hline Legumes & 0.06 & 0.43 & 0.19 & -0.01 \\
\hline Sugared drinks $†$ & 0.48 & 0.04 & -0.18 & -0.29 \\
\hline Whole grains & 0.31 & 0.37 & 0.21 & 0.03 \\
\hline Pizza & 0.56 & -0.23 & -0.18 & 0.28 \\
\hline Burgers & 0.48 & -0.19 & -0.24 & 0.28 \\
\hline Souvlaki & 0.54 & -0.20 & -0.16 & 0.24 \\
\hline Chips/crisps & 0.52 & -0.23 & 0.06 & -0.17 \\
\hline French fries & 0.56 & -0.09 & 0.02 & -0.01 \\
\hline Fish & 0.29 & 0.39 & -0.12 & 0.01 \\
\hline Honey/jam & 0.33 & 0.42 & 0.10 & $0 \cdot 11$ \\
\hline Sweetsł & 0.64 & -0.12 & $0 \cdot 12$ & 0.04 \\
\hline Ordering/eating out & 0.47 & -0.29 & -0.08 & 0.22 \\
\hline Number of eating occasions & 0.20 & -0.01 & 0.69 & 0.07 \\
\hline Having meal while watching TV or playing video games & 0.42 & -0.28 & 0.37 & -0.31 \\
\hline Breakfast frequency & 0.04 & 0.39 & 0.37 & 0.46 \\
\hline Screen time & 0.26 & -0.31 & 0.31 & -0.35 \\
\hline Family meals frequency & 0.04 & 0.32 & 0.01 & 0.33 \\
\hline
\end{tabular}

TV, television; SSB, sugar-sweetened beverages.

Coefficients with absolute loadings $>0.4$ are indicated in bold font

${ }^{*}$ Consumption of home-made and market/packaged $100 \%$ fruit juice.

†Consumption of soft drinks and sugared fruit juices (SSB).

$\ddagger$ Consumption of chocolate, chocolate bars, ice cream and pastries.

together contributed $2.6 \%$ of total energy, breakfast cereals $2.7 \%$, biscuits $1.4 \%$, starchy pies $1.7 \%$ and honey/ jam less than $1 \%$.

Based on factor analysis, four components were extracted and studied that explained $50 \%$ of the total variation with regard to the examined variables. The value of the Kaiser-Meyer-Olkin criterion was equal to 0.8 and the $P$ value for Bartlett's test of sphericity was $<0.001$, indicating that the dietary variables entered in the analysis were strongly inter-correlated and, therefore, factor analysis could be used for assessing meaningful dietary patterns. The loadings for the four food components (patterns), which represent the correlation of each food item with the corresponding component, are presented in Table 3 (bold font indicates the coefficients with absolute loadings $>0 \cdot 4$, which means they are better correlated with the component). Since higher absolute values indicate that the food variable contributes more to the characterization of the component ${ }^{(32)}$, it could be suggested that the extracted components are characterized as follows: higher 
consumption of sweets, pizza, souvlaki, burgers, French fries and sugared drinks, more frequently ordering/eating outside home and having meals in front of a screen (component 1); higher consumption of whole fruits, $100 \%$ natural fruit juices, vegetables, legumes and honey/jam (component 2); a pattern loaded heavily on daily number of meals and snacks (eating occasions; component 3); and a pattern characterized mainly by breakfast frequency (component 4).

Potential associations between the extracted lifestyle patterns and free sugars intake were explored by multiple linear regression analysis, after controlling for sex, age and energy intake. As shown in Table 4, lifestyle patterns 1 and 2 , and children's age were positively associated with free sugars intake. Conversely, lifestyle patterns 3 and 4 were negatively associated with free sugars intake. Lastly, the evaluation of the association between free sugars intake and OW/OB with the logistic regression analysis showed that free sugars intake was not a significant predictor of $\mathrm{OW} / \mathrm{OB}$ after adjusting for age, sex, physical activity level and energy intake (Table 5).

Table 4 Multiple linear regression analysis examining the association of lifestyle components with free sugars intake in a nationwide sample of 3089 children (aged 10-12 years), GRECO (Greek Childhood Obesity) study, 2009

\begin{tabular}{|c|c|c|}
\hline Variable & $\beta$ & $P$ value \\
\hline Female & -0.02 & 0.11 \\
\hline Age (years) & 0.03 & 0.04 \\
\hline Energy intake (kcal/d) & 0.26 & $<0.001$ \\
\hline $\begin{array}{l}\text { Higher consumption of fast foods and sweets, } \\
\text { increased screen time and frequent eating out* }\end{array}$ & 0.52 & $<0.001$ \\
\hline $\begin{array}{l}\text { Higher consumption of whole fruits, } 100 \% \text { natural } \\
\text { fruit juices, vegetables, legumes and honey/jam }\end{array}$ & $0 \cdot 18$ & $<0.001$ \\
\hline Higher daily number of meals and snacks $\ddagger$ & -0.14 & $<0.001$ \\
\hline Higher breakfast frequency§ & -0.18 & $<0.001$ \\
\hline \multicolumn{3}{|c|}{$\begin{array}{l}\beta \text {, standardized beta coefficient. } \\
\text { *Lifestyle component } 1 \text { : higher consumption of sweets, pizza, souvlaki, } \\
\text { burgers, French fries and sugared drinks, more frequently ordering/eating } \\
\text { outside home and having meals in front of a screen. } \\
\text { tLifestyle component 2: higher consumption of whole fruits, } 100 \% \text { natural } \\
\text { fruit juices, vegetables, legumes and honey/jam. } \\
\text { fLifestyle component } 3 \text { : higher daily number of meals and snacks. } \\
\text { §Lifestyle component } 4: \text { higher breakfast frequency. }\end{array}$} \\
\hline
\end{tabular}

\section{Discussion}

The present study is the first estimating free sugars intake in a Greek childhood population. Adopting the WHO recommendations ${ }^{(19)}$, it was shown that a large proportion of children exceeded the cut-off of $10 \%$ of energy coming from free sugars. The overall mean contribution of free sugars to total energy intake was $11 \cdot 2 \%$, lower than the percentage reported for the child population in the USA (16.2\% for children $6-11$ years old and $17 \%$ for adolescents $12-19$ years old $)^{(10)}$, but similar to other childhood populations ${ }^{(33,34)}$. It should be stressed though that it is difficult to compare intakes among different populations since there is no universally accepted definition of the term 'added sugars' and a lack of agreement on the foods that contribute to added sugars intake. According to Slining and Popkin ${ }^{(35)}$ who examined 15-year trends in intakes and sources of added sugars among US children aged 2-18 years, the intake of added sugars as a percentage of total energy intake declined significantly from $18 \%$ in 1994-1998 to $14 \%$ in 2009-2010, together with the average daily energy intake from added sugars. According to those authors, although the results highlighted a recent decline in intake, they also showed that the average US child still consumes a great amount of energy from added sugars. The main sources of added sugar intake, according to the same report, were SSB, grain-based desserts, candy and other sweet snacks, ready-to-eat cereals, dairy-based desserts, and sweeteners and syrups. Similar findings regarding the major sources of added sugars have been reported for US children, with SSB being the primary source $^{(10,36)}$.

The analysis of the present study revealed a considerably different scenario with $100 \%$ packaged and freshly squeezed fruit juice being the principal source of free sugars, followed by sweets, nectar (sugared) fruit juices, chocolate milk, soft drinks and other foods presented in Table 1. Besides revealing variations in food consumption habits and preferences between childhood populations, these differences also bring to light a serious methodological problem when investigating the

Table 5 Results from logistic regression analysis to evaluate the main effect of child variables on the likelihood of childhood overweight/obesity in a nationwide sample of 3089 children (aged 10-12 years), GRECO (Greek Childhood Obesity) study, 2009

\begin{tabular}{|c|c|c|c|c|}
\hline & \multicolumn{2}{|c|}{ Model 1} & \multicolumn{2}{|c|}{ Model 2} \\
\hline & OR & $95 \% \mathrm{Cl}$ & OR & $95 \% \mathrm{Cl}$ \\
\hline \multicolumn{5}{|l|}{ Sex } \\
\hline Female & 1.00 & Ref. & 1.00 & Ref. \\
\hline Male & $1 \cdot 14$ & $0.91,1.44$ & $1 \cdot 12$ & $0.89,1.42$ \\
\hline Age & 0.77 & $0.66,0.91$ & 0.77 & $0.66,0.91$ \\
\hline PAQ-C score & 0.87 & $0.72,1.05$ & 0.87 & $0.72,1.05$ \\
\hline Free sugars intake (per $10 \mathrm{~g}$ ) & 0.98 & $0.96,1.01$ & 0.97 & $0.94,1.01$ \\
\hline Energy intake (per $418 \mathrm{~kJ}(100 \mathrm{kcal}))$ & - & - & 1.10 & $1.08,1.13$ \\
\hline
\end{tabular}

PAQ-C, Physical Activity Questionnaire for Older Children; Ref., referent category.

All odds ratios and their corresponding $95 \%$ confidence intervals were calculated by performing multiple logistic regressions. 
association of sugars or added sugars intake with $\mathrm{OB}$ or other health outcomes, since there are important differences between studies concerning the inclusion or exclusion of foods that are considered or not sources of added (or free) sugars. The percentage of energy coming from soft drinks and sugared fruit juices in our study was lower than the respective proportions found in other studies where it has been shown that energy intake from sodas and from all SSB account for 5.8\% and $8.5 \%$, respectively ${ }^{(37)}$. In further studies and depending on the grouping of the foods prior to the analyses, it has been reported that children and adolescents consume 10-15\% of energy intake from SSB and $100 \%$ fruit juice ${ }^{(37,38)}$.

Irrespective of the finding that added sugars intake seems to have levelled off in some populations, it still remains higher than the recommended upper intake levels ${ }^{(35,39)}$. An array of meta-analyses have shown a significant association between sugar intake (generally focused on sugars from SSB) and OB or diabetes mostly in adults but in children as well ${ }^{5,39-41)}$. Furthermore, intervention studies in children and adolescents have shown that reducing SSB consumption significantly reduces weight gain and adiposity ${ }^{(42,43)}$. However, there are also prospective and cross-sectional studies showing no association or even a negative association between sugars intake and body weight ${ }^{(14-16)}$. In the study of Song et $a l{ }^{(16)}$ it was shown that energy intake rather than total sugar intake was the most significant dietary factor predicting BMI in children and adolescents. The former study highlighted that the determining factor influencing OW and $\mathrm{OB}$ rates is ultimately the imbalance between energy intake and energy expenditure rather than the consumption of a specific nutrient per se. In the present study we found no differences in BMI or any other central obesity index between children exceeding or not the cut-off of $10 \%$ of energy coming from free sugars. Furthermore, logistic regression analysis (Table 5) showed that free sugars intake was not a significant predictor of $\mathrm{OW} / \mathrm{OB}$, while, as expected, higher energy intake increased the odds for OW/OB. A possible explanation for the lack of association between free sugars and OW/OB could be that the highest proportion of energy intake in the diet of Greek children came from foods and food groups that do not contain free sugars (Fig. 1).

In the present study, $44 \%$ of children exceeded the cutoff of $10 \%$ of energy coming from free sugars when using the recommendation of the WHO, with the primary source of free sugars being $100 \%$ packaged and freshly squeezed fruit juice. Following the American Academy of Pediatrics recommendation, public health authorities in many countries suggest the limitation of fruit juice intake to approximately $237-355 \mathrm{ml} / \mathrm{d}(8-12 \mathrm{fl} \mathrm{oz} / \mathrm{d}$ ) in children and adolescents 7 to 18 years old, mostly led by the concern that high fruit juice consumption is associated with OW in children $^{(44,45)}$. Nevertheless, this recommendation is disputed by other researchers mostly because several studies examining the long-term effects of fruit juice consumption find no effect or adverse effect on the body weight of children $^{(46-48)}$. In addition, it has been stressed that some food sources of added sugars (including $100 \%$ fruit juices) make a major contribution to micronutrient and fibre intakes in the overall diet and that consumers of $100 \%$ fruit juice show better nutrient adequacy and diet quality than non-consumers ${ }^{(36,49)}$.

The free sugars intake was tested in relation to lifestyle patterns of children. Two of the patterns extracted by factor analysis, characterized by higher consumption of sweets, pizza, souvlaki, burgers, French fries and sugared drinks, more frequently ordering/eating outside home and having meals in front of a screen (component 1) and higher consumption of whole fruits, $100 \%$ natural fruit juices, vegetables, legumes and honey/jam (component 2), were positively associated with free sugars intake. Pattern analysis as a method has the advantage of revealing behaviours in populations that could not be assessed with any other traditional dietary assessment method and gives the opportunity to examine the effects of overall dietary choices and lifestyle characteristics on outcomes in a more integrated way ${ }^{(22)}$. In the extracted component 1 , one of the loadings was SSB consumption which has been independently associated with increased consumption of added sugars $^{(10,35,36)}$. However, the component reveals a whole unfavourable lifestyle, instead of one food, that promotes free sugars intake and includes regular consumption of sweets and fast foods, eating/ordering outside home and having meals in front of a screen. High fast-food intake, eating out and having meals in front of a screen in adolescents have been associated with unfavourable changes in dietary habits and BMI, and increased consumption of foods high in added sugars ${ }^{(50-52)}$. Furthermore, it has been suggested that the negative association between added sugars intake and micronutrient density of diets is more related to patterns of food intakes from which added sugars in the diet are derived, rather than to added sugars intake per $s e^{(3)}$. In the case of component 2 , which was also positively associated with free sugars intake, it is mainly characterized by foods that are considered healthy and are generally promoted to be consumed regularly. The association of this particular component with free sugars intake could be mainly attributed to the fact that it is loaded by $100 \%$ fruit juice and honey/jam. It could be hypothesized that both $100 \%$ fruit juice and honey/jam are considered healthy choices by the children and their parents. Previous reports also show that fruit juice is considered to be a 'healthy' beverage and most studies of dietary intake patterns associated with an otherwise healthy diet among children find fruit juice as the main beverage parents provide their children ${ }^{(53)}$. Finally, the two patterns characterized mainly by higher daily number of meals and snacks (component 3) and higher breakfast frequency (component 4) showed a negative association with free sugars intake. Previous work in this area has shown that both frequent breakfast 
consumption and a higher number of meals during the day, besides protecting against childhood OW/OB, are also associated with better diet quality ${ }^{(54-57)}$.

The limitations of the present work are mostly due to its cross-sectional nature since cause-and-effect relationships cannot be drawn. The use of international instead of local food composition tables (due to the incompleteness) may have over- or underestimated the energy and free sugars content of some of the foods included in the analyses, although we have used the NDSR software which provides data on free sugars for a large variety of foods. Additionally, we must highlight that research suggests that collecting reliable and accurate dietary data from children and adolescents is difficult since under-reporting appears to be greatest for unhealthy foods or foods perceived to be related to $\mathrm{OB}$, such as those high in added sugars ${ }^{(35)}$. In the present study, we identified under-reporting children but decided not to exclude them from the analyses since we did not find differences in the percentage of under-reporters and normal reporters exceeding the limit of $10 \%$ of total energy coming from free sugars and when examining the main sources of free sugars, total sugars and energy in the diet of under- and normal-reporting children, no differences were observed in the classification of food sources. Furthermore, when excluding under-reporters we did not find differences in the associations from the logistic regression analysis evaluating the effect of variables on the likelihood of childhood OW/OB.

\section{Conclusion}

The present study reveals that a high proportion of Greek children exceed the current WHO recommendation for free sugars intake. Although it is not clear in the literature what the mechanisms for producing adverse effects on BMI or cardiometabolic factors from increased added sugars intake are, there is a consensus that high free (or added) sugars intake increases the risk for adverse health outcomes through high energy intake. Thus, just like any other nutrient, free sugars should have an upper limit of intake. However, the food sources of free sugars in Greek children were different from those identified in other populations, and more importantly free sugars intake was associated with lifestyle patterns rather than single foods. One of these patterns revealed that frequent ordering out, consumption of fast foods, as well as having meals in front of a screen were associated with increased consumption of foods high in free sugars and consequently higher total free sugars intake. On the other hand, a pattern characterized by consumption of healthy foods (such as whole fruits, $100 \%$ fruit juice, honey, vegetables and legumes) was also related to higher free sugars intake. Therefore, before characterizing foods as 'good' or 'bad' based only on their concentration of free sugars we have to consider the contribution of every food or dietary pattern to overall diet and health. Any public health initiatives aiming to reduce free sugars intake should focus on promoting the correct or altering the lifestyle habits of the specific childhood population.

\section{Acknowledgements}

Financial support: Funding for the study was provided by the General Secretariat of Consumers-Greek Ministry of Development, Hellenic Association of Food and Beverage Companies, Coca-Cola Hellas, Coca-Cola Hellenic Bottling Company, Cereal Partners Hellas, FAGE SA, Unilever Hellas, Nestlé Hellas and Kraft Foods Hellas. This research has also been co-financed by the European Union (European Social Fund - ESF) and Greek national funds through the Operational Program 'Education and Lifelong Learning' of the National Strategic Reference Framework (NSRF) - Research Funding Program: 'Heraclitus II. Investing in knowledge society through the European Social Fund'. All mentioned funders had no role in the design, analysis or writing of this article. Conflict of interest: None. Authorship: A.Z., D.B.P., P.F. and G.R. were responsible for the study design and the supervision of the field study. D.B.P. and P.F. were responsible for the statistical analysis. P.F., D.B.P., G.R. and A.Z. were responsible for the interpretation of the data. All authors carried out data collection or data management, contributed to database preparation and participated in writing the submitted manuscript. All authors read and approved the final manuscript. Ethics of human subject participation: This study was conducted according to the guidelines laid down in the Declaration of Helsinki and all procedures involving human subjects were approved by the Hellenic Ministry of Education (Department of Primary Education) and the Agricultural University of Athens Research Committee.

\section{References}

1. Johnson R, Appel L, Brands M et al. (2009) Dietary sugars intake and cardiovascular health. A scientific statement from the American Heart Association. Circulation 120, 1011-1020.

2. Murphy S \& Johnson R (2003) The scientific basis of recent US guidance on sugars intake. Am J Clin Nutr 78, issue 4, 827S-833S.

3. Hess J, Latulippe ME, Ayoob K et al. (2012) The confusing world of dietary sugars: definitions, intakes, food sources and international dietary recommendations. Food Funct 3, 477-486.

4. Anderson CA, Curzon ME, Van Loveren C et al. (2009) Sucrose and dental caries: a review of the evidence. Obes Rev 10, 41-54.

5. Te Morenga L, Mallard S \& Mann J (2012) Dietary sugars and body weight: systematic review and meta-analyses of randomised controlled trials and cohort studies. BMJ $\mathbf{3 4 6}$, e7492. 
6. Lobstein T \& Frelut ML (2004) Prevalence of overweight among children in Europe. Obes Rev 4, 195-200.

7. Ogden CL, Carroll MD, Kit BK et al. (2012) Prevalence of obesity and trends in body mass index among US children and adolescents, 1999-2010. JAMA 307, 483-490.

8. Farajian P, Risvas G, Karasouli K et al. (2011) Very high childhood obesity prevalence and low adherence rates to the Mediterranean diet in Greek children: the GRECO study. Atherosclerosis 217, 525-530.

9. Moschonis G, Kalliora AC, Costarelli V et al. (2014) Identification of lifestyle patterns associated with obesity and fat mass in children: the Healthy Growth study. Public Health Nutr 17, 614-624.

10. Drewnowski A \& Rehm CD (2014) Consumption of added sugars among US children and adults by food purchase location and food source. Am J Clin Nutr 100, 901-907.

11. Lee AK, Binongo JN, Chowdhury R et al. (2014) Consumption of less than $10 \%$ of total energy from added sugars is associated with increasing HDL in females during adolescence: a longitudinal analysis. J Am Heart Assoc 26, e000615.

12. Welsh JA, Sharma A, Cunningham SA et al. (2011) Consumption of added sugars and indicators of cardiovascular disease risk among US adolescents. Circulation 123, 249-257.

13. Welsh JA, Sharma A, Abramson JL et al. (2010) Caloric sweetener consumption and dyslipidemia among US adults. JAMA 303, 1490-1497.

14. Ludwig D, Peterson K \& Gortmaker S (2001) Relation between consumption of sugar-sweetened drinks and childhood obesity: a prospective, observational analysis. Lancet 357, 505-508.

15. Chun OK, Chung CE, Wang Y et al. (2010) Changes in intakes of total and added sugars and their contribution to energy intake in the US. Nutrients 2, 834-835.

16. Song WO, Wang Y, Chung CE et al. (2012) Is obesity development associated with dietary sugar intake in the US? Nutrition 28, 1137-1141.

17. European Food Safety Authority Panel on Dietetic Products, Nutrition and Allergies (2010) Scientific opinion on dietary reference values for carbohydrates and dietary fibre. EFSAJ 8, 1462 .

18. Institute of Medicine, Food and Nutrition Board (2002) Dietary Reference Intakes for Energy, Carbohydrate, Fiber, Fat, Fatty Acids, Cholesterol, Protein, and Amino Acids. Washington, DC: National Academies Press.

19. World Health Organization (2003) Diet, Nutrition, and the Prevention of Chronic Diseases. Joint WHO/FAO Expert Consultation. WHO Technical Report Series no. 916. Geneva: WHO.

20. Jacques PF \& Tucker KL (2001) Are dietary patterns useful for understanding the role of diet in chronic disease? $A m \mathrm{~J}$ Clin Nutr 73, 1-2.

21. Trichopoulos D \& Lagiou P (2001) Dietary patterns and mortality. Br J Nutr 85, 133-134.

22. Kim J-O \& Mueller C (1978) Factor Analysis: Statistical Methods and Practical Issues. Newbury Park, CA: SAGE Publications, Inc.

23. Farajian P, Panagiotakos DB, Risvas G et al. (2013) Socioeconomic and demographic determinants of childhood obesity prevalence in Greece: the GRECO (Greek Childhood Obesity) study. Public Health Nutr 16, 240-247.

24. Bountziouka V, Farajian P, Risvas G et al. (2013) Development and validation of a semi-quantitative food frequency questionnaire for young school-aged children. Ann Nutr Metab 63, 1-1960 (abstr.).

25. Farajian P, Karasouli K, Risvas G et al. (2009) Repeatability and validity of a food frequency and dietary habits questionnaire in children. Circulation 119, e288 (abstr.).
26. Schakel SF, Sievert YA \& Buzzard IM (1998) Sources of data for developing and maintaining a nutrient database. $J$ Am Diet Assoc 88, 1268-1271.

27. Black A (2000) Critical evaluation of energy intake using the Goldberg cut-off for energy intake:basal metabolic rate. A practical guide to its calculation, use and limitations. Int $J$ Obes Relat Metab Disord 24, 1119-1130.

28. Schofield WN (1985) Predicting basal metabolic rate, new standards and review of previous work. Hum Nutr Clin Nutr 39C, 5-41.

29. Nelson M, Black AE, Morris JA et al. (1989) Between- and within-subject variation in nutrient intake from infancy to old age: estimating the number of days required to rank dietary intakes with desired precision. Am J Clin Nutr 50, 155-167.

30. Cole TJ, Bellizzi MC, Flegal KM et al. (2000) Establishing a standard definition for child overweight and obesity worldwide: international survey. BMJ 320, 1240-1243.

31. Kowalski KC, Crocker PRE \& Faulkner RA (1997) Validation of the physical activity questionnaire for older children. Pediatr Exerc Sci 9, 174-186.

32. Mardia KV, Kent JT \& Bibby JM (1979) Multivariate Analysis, 1st ed. New York: Academic Press.

33. Brisbois TD, Marsden SL, Anderson GH et al. (2014) Estimated intakes and sources of total and added sugars in the Canadian diet. Nutrients 6, 1899-1912.

34. Colucci AC, Cesar CL, Marchioni DM et al. (2012) Factors associated with added sugars intake among adolescents living in São Paulo, Brazil. J Am Coll Nutr 31, 259-267.

35. Slining MM \& Popkin BM (2013) Trends in intakes and sources of solid fats and added sugars among US children and adolescents: 1994-2010. Pediatr Obes 8, 307-324.

36. Huth PJ, Fulgoni VL, Keast DR et al. (2013) Major food sources of calories, added sugars, and saturated fat and their contribution to essential nutrient intakes in the US diet: data from the National Health and Nutrition Examination Survey (2003-2006). Nutr J 12, 116.

37. Reedy J \& Krebs-Smith SM (2010) Dietary sources of energy, solid fats, and added sugars among children and adolescents in the United States. J Am Diet Assoc 110, $1477-1484$.

38. Wang YC, Bleich SN \& Gortmaker SL (2008) Increasing caloric contribution from sugar-sweetened beverages and $100 \%$ fruit juices among US children and adolescents, 1988-2004. Pediatrics 121, e1604-e1614.

39. Bray GA \& Popkin BM (2014) Dietary sugar and body weight: have we reached a crisis in the epidemic of obesity and diabetes? Health be damned! Pour on the sugar. Diabetes Care 37, 950-956.

40. Malik VS, Schulze MB \& Hu FB (2006) Intake of sugarsweetened beverages and weight gain: a systematic review. Am J Clin Nutr 84, 274-288.

41. Hu FB (2013) Resolved: there is sufficient scientific evidence that decreasing sugar-sweetened beverage consumption will reduce the prevalence of obesity and obesity-related diseases. Obes Rev 14, 606-619.

42. Ebbeling CB, Feldman HA, Chomitz VR et al. (2012) A randomized trial of sugar-sweetened beverages and adolescent body weight. $N$ Engl J Med 367, 1407-1416.

43. de Ruyter JC, Olthof MR, Seidell JC et al. (2012) A trial of sugar-free or sugar-sweetened beverages and body weight in children. N Engl J Med 367, 1397-1406.

44. American Academy of Pediatrics (2001) The use and misuse of fruit juice in pediatrics. Pediatrics 107, 1210-1213.

45. Dennison BA, Rockwell HL \& Baker SL (1997) Excess fruit juice consumption by pre-school-aged children is associated with short stature and obesity. Pediatrics 99, 15-22.

46. Skinner JD, Carruth BR, Moran J 3rd et al. (1999) Fruit juice intake is not related to children's growth. Pediatrics 103, 58-64. 
47. Alexy U, Sichert-Hellert W, Kersting M et al. (1999) Fruit juice consumption and the prevalence of obesity and short stature in German preschool children: results of the DONALD study. Dortmund nutritional and anthropometrical longitudinally designed. J Pediatr Gastroenterol Nutr 29, 343-349.

48. Newby PK, Peterson KE, Berkey CS et al. (2004) Beverage consumption is not associated with changes in weight and body mass index among low-income preschool children in North Dakota. J Am Diet Assoc 104, 1086-1094.

49. O'Neil CE \& Nicklas TA (2014) Childhood obesity and the consumption of $100 \%$ fruit juice: where are the evidencebased findings? In Fructose, High Fructose Corn Syrup, Sucrose and Health, pp. 247-275 [JM Rippe, editor]. New York: Springer.

50. Gebremariam MK, Bergh IH, Andersen LF et al. (2013) Are screen-based sedentary behaviors longitudinally associated with dietary behaviors and leisure-time physical activity in the transition into adolescence? Int J Behav Nutr Phys Act 10,9 .

51. Olafsdottir S, Berg C, Eiben G et al. (2014) Young children's screen activities, sweet drink consumption and anthropometry: results from a prospective European study. Eur J Clin Nutr 68, 223-228.
52. Demissie Z, Eaton DK, Lowry R et al. (2014) The association of meal practices and other dietary correlates with dietary intake among high school students in the United States, 2010. Am J Health Promot 29, e203-e213.

53. Piernas C, Tate DF, Wang X et al. (2013) Does diet-beverage intake affect dietary consumption patterns? Results from the Choose Healthy Options Consciously Everyday (CHOICE) randomized clinical trial. Am J Clin Nutr 97, 604-611.

54. Deshmukh-Taskar PR, Nicklas TA, O'Neil CE et al. (2010) The relationship of breakfast skipping and type of breakfast consumption with nutrient intake and weight status in children and adolescents: the National Health and Nutrition Examination Survey 1999-2006. J Am Diet Assoc 110, 869-878.

55. Farajian P, Panagiotakos DB, Risvas G et al. (2014) Hierarchical analysis of dietary, lifestyle and family environment risk factors for childhood obesity: the GRECO study. Eur J Clin Nutr 68, 1107-1112.

56. Nicklas TA, Myers L, Reger C et al. (1998) Impact of breakfast consumption on nutritional adequacy of the diets of young adults in Bogalusa, Louisiana: ethnic and gender contrasts. J Am Diet Assoc 98, 1432-1438.

57. Nicklas TA, Baranowski T, Cullen KW et al. (2001) Eating patterns, dietary quality and obesity. J Am Coll Nutr 20, 599-608. 